\title{
Production of photons from a chemically non equilibrating plasma in relativistic heavy-ion collisions
}

\section{Yogesh Kumar*}

Department of Physics, Sri Aurobindo College, University of Delhi, New Delhi-110017, India E-mail: vogesh.du81egmail.com

\section{Poonam Jain}

Department of Physics, Sri Aurobindo College, University of Delhi, New Delhi-110017, India E-mail: poonam.jniegmail.com

\begin{abstract}
We investigate the photon radiation of leading order process in collisions of two heavy nuclei using various value of quark phenomenological flow parameter dependent on finite value of quark chemical potential in quark mass. The photon emission rate is observed using various initial condition taken from HIJING calculations for gold-gold collisions at RHIC and LHC energies. Our results for photon measurement is strongly increasing function of quark flow parameter ranging $2 \gamma_{g} \leq \gamma_{q} \leq 8 \gamma_{g}$ and give the significant contribution at higher temperature and quark chemical potential.
\end{abstract}

9th International Workshop on Critical Point and Onset of Deconfinement - CPOD2014,

17-21 November 2014

ZiF (Center of Interdisciplinary Research), University of Bielefeld, Germany

\footnotetext{
* Speaker.
} 


\section{Introduction}

The theoretical and experimental investigation of quark gluon plasma (QGP) under extreme conditions has become an exciting challenges of high energy physics at high temperature and baryon density [四]. Such a new state of matter can be produced in these experiments by collid-

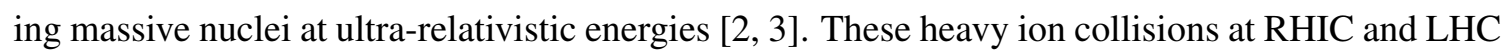
thus provide the exact information for the detection and formation of baryon rich QGP [ [⿴囗十, 6, 因]. Researcher believe that the detection of QGP is very difficult due to its short time visibility. There are many indirect probes for detection of QGP [Q, [ 8$]$ and among all proposed probes, photons are regarded as potential probes of the dynamics of nucleus nucleus reactions [Q $[0, \mathbf{0}, \mathbf{W}]$. Because of weakly interacting nature, they emit continuously at every point in the space time evolution of fireball. These particles are sensitive probes to the dynamics of the deconfined phase. The experiments such as WA98 at SPS/CERN [ए2] and PHENIX at RHIC/BNL [ए3] have showed explicit data points for direct photons.

So far authors have studied the photon production from a QGP [14, [5], [16, [17, [8]. Nagamiya has considered a finite value of chemical potential by assuming the formation of QGP already at AGS and SPS energies [प्प]. Infact the value of chemical potential cannot be ignored as the plasma is not fully transparent in the central collision of massive nuclei and it has some finite value at RHIC energies. Thus the baryon density may be large in the QGP formation and then thermodynamic equilibrium is a function of both the temperature and the chemical potential. In recent past, the calculation for QGP phase is not expected to be in chemical equilibrium [201, 221, [22]. Further Strickland, Traxler and their co-workers has been estimated the photon spectrum from a chemically equilibrating baryon free QGP system [23], 24]].

The authors [25, [26] have been calculated the photon yield from a chemically equilibrated baryon rich plasma. In addition, the significant contribution for calculating the photon production from a chemically equilibrating plasma at finite baryon density has been discussed by Ref. [27, 28], [29, [30]. This would lead to the modification of photon emission rates. In this work, we study the production of photons from a chemically non-equilibrated plasma with variation of flow parameters at finite chemical potential using various initial condition taken from HIJING calculations for goldgold collisions at RHIC and LHC energies [23]].

In this article, we use a simple phenomenological model for calculating leading order process from quark-gluon plasma (QGP) using various value of quark phenomenological flow parameter dependent on finite quark chemical potential in quark mass. Here, quark mass is dependent on temperature and chemical potential using parametrization factors [B], B2]. The mass of quark shows well behaviour above critical temperature. The finite quark mass is defined as [32, [33, [34, [3]]:

$$
m_{q}^{2}(T, \mu)=\gamma_{q, g}^{\prime}\left(\lambda_{g}+\frac{\lambda_{q}}{2}\right) g^{2}(k) T^{2} .
$$

where $\gamma_{q, g}^{\prime}$ is the phenomenological parameter of quarks and gluons, $k$ is the quark (gluon) momentum, T is the temperature and $g(k)$ is first order QCD running coupling constant taken by [B4, [3]]. The parameter $\gamma_{q}^{\prime}$ is taken by modifying the earlier value of $\gamma_{q}$. We replace $\gamma_{q}$ by $\gamma_{q}[1+$ $\mu_{q}^{2} / \pi^{2} T^{2}$ ] [33]. We use this modified value as $\gamma_{q}^{\prime}$ [33], [36]. On the other side, we put gluon parameter remain same i.e. $\gamma_{g}^{\prime}$ as $\gamma_{g}$. The value of $\gamma_{g}$ is fixed as $1 / 3$ given in the Ref. [3]]. The 
parametrization factor $\gamma^{2}=2\left[\frac{1}{\gamma_{q}^{2}}+\frac{1}{\gamma_{g}^{2}}\right]$. We fix the range of $\gamma_{q}$ as $\gamma_{q}=2 \gamma_{g}$ to $8 \gamma_{g}$ and $\gamma_{g}=1 / 3$ [B]] in our calculation. We compute the photon emission from non-equilibrated plasma of complete leading order (LO) results using HIJING initial condition at RHIC and LHC energies at temperatures $T=0.57 \mathrm{GeV}$ and $0.83 \mathrm{GeV}$ with the higher value of quark chemical potential for flavor 3 .

Thus, we organize the paper as follows: In section $I I$, we give brief outline of LO process for photon spectra from QGP. In section $I I I$, we present the results and at last section $I V$ we give the conclusion.

\section{Production of photons from QGP}

The complete calculation of thermal photons from a partonic medium to order $\alpha_{s}$ has been done in the Ref. [144, 37, 38, [3]]. We extend our work at a finite temperature and high chemical potential from a chemically non-equilibrated QGP system using various set of initial condition at RHIC and LHC energies.

The complete calculation of photon rate of momentum $k$ up to two loop is given by the expression [30, 40]

$$
\frac{d n}{d^{4} x d^{3} k}=\frac{1}{(2 \pi)^{3}} A(k)\left[\ln \left(\frac{T}{m_{q}(T, \mu)}\right)+\frac{1}{2} \ln \left(\frac{2 k}{T}\right)+C_{t o t}\left(\frac{k}{T}\right)\right]
$$

with $m_{q}^{2}(T, \mu)$ is the leading order large momentum limit of the quark mass. The leading-log coefficient $A(k)$ is given as

$$
A(k)=4 \alpha_{e} \frac{m_{q}^{2}(T, \mu)}{k} F_{D}(k)
$$

where $\alpha_{e}$ is the electromagnetic constant and mass of quark is taken by Ref. [B3], [34]. $F_{D}(k)$ is the Fermi distribution function. The dependence on the specific photon production process is written in the term $C_{\text {tot }}\left(\frac{k}{T}\right)$,

$$
C_{\text {tot }}\left(\frac{k}{T}\right)=C_{2 \leftrightarrow 2}\left(\frac{k}{T}\right)+C_{\text {brems }}\left(\frac{k}{T}\right)+C_{\text {aws }}\left(\frac{k}{T}\right) .
$$

The $C_{t o t}\left(\frac{k}{T}\right)$ is the non-trivial function that can only be solved numerically. The results of $C_{t o t}\left(\frac{k}{T}\right)$ is taken by Ref. [39, 40]].

Then we study the total photon spectrum above critical temperature by integrating the total rate over the space-time history of the collision. It is expressed as [231, 40, 41], 42, 43], 44, 45]]:

$$
\begin{gathered}
\frac{d n}{d^{2} k_{T} d y}=\int d^{4} x\left(k \frac{d n}{d^{3} k d^{4} x}\right) \\
=Q \int_{\tau_{0}}^{\tau_{f}} \tau d \tau \int_{-y_{\text {nuc }}}^{y_{\text {nuc }}} d y\left(k \frac{d n}{d^{3} k d^{4} x}\right) .
\end{gathered}
$$

With various set of HIJING initial condition for gold-gold collisions, the following parameters are set as fugacity for quark and gluon are $\lambda_{q}=0.02, \lambda_{g}=0.09$, rapidity of nuclei is $y_{n u c}=6$, initial to final times are $\tau_{0}=0.7 \mathrm{fm}$ to $\tau_{f}=4 \mathrm{fm}$ and $T_{0}=0.57 \mathrm{GeV}$ at RHIC energy. While fugacity for quark and gluon are $\lambda_{q}=0.03, \lambda_{g}=0.14$, rapidity of nuclei is $y_{\text {nuc }}=8.8$, initial to final times are $\tau_{0}=0.5 \mathrm{fm}$ to $\tau_{f}=6.25 \mathrm{fm}$ and $T_{0}=0.83 \mathrm{GeV}$ are taken at LHC energy. The transverse cross section of the gold nuclei; $\mathrm{Q} \approx 180 \mathrm{fm}^{2}$. Using all these parameters, we obtain the total photon spectrum at RHIC and LHC. 


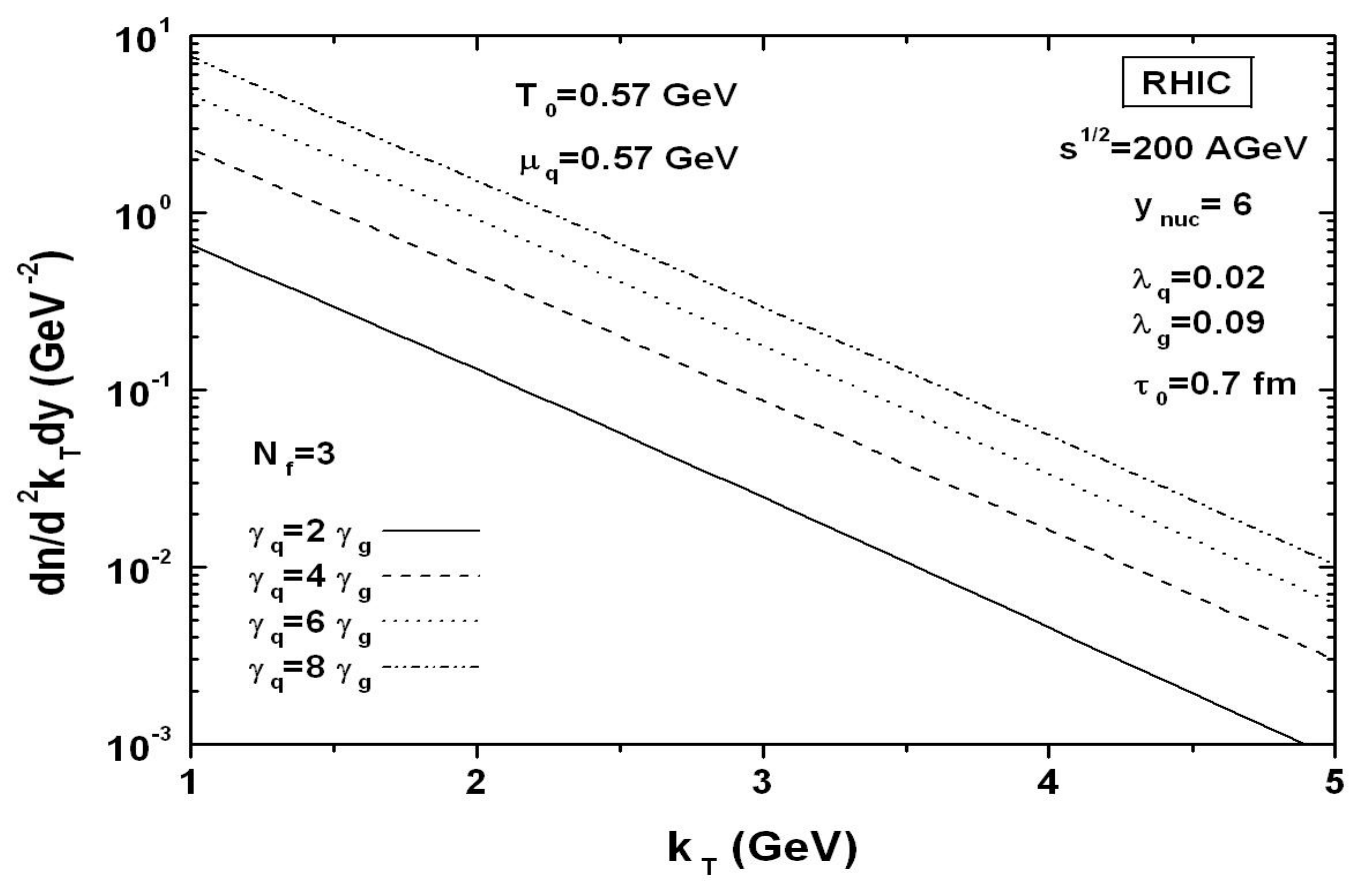

Figure 1: The photon spectra at thermal temperature $T_{0}=0.57 \mathrm{GeV}$ for $N_{f}=3$ with the finite value of quark chemical potential $\mu_{q}=0.57 \mathrm{GeV}$ with fix value of $\gamma_{g}=1 / 3$ and various value of parameter range from $\gamma_{q}^{\prime}=2\left[1+\mu_{q}^{2} / \pi^{2} T^{2}\right] \gamma_{g}$ to $8\left[1+\mu_{q}^{2} / \pi^{2} T^{2}\right] \gamma_{g}$ i.e. $\gamma_{q}=2 \gamma_{g}$ to $8 \gamma_{g}$ using HIJING initial condition at RHIC.

\section{Results}

We study the photon production of leading order process using various initial condition of HIJING calculation for gold-gold collisions at RHIC and LHC with the effect of phenomenological parameters such as $\gamma_{q}^{\prime}$ and $\gamma_{g}$ dependent on quark chemical potential in quark mass. The calculation of LO processes for photon emission is extended with the suitable range of phenomenological parameter from $\gamma_{q}=2 \gamma_{g}$ to $8 \gamma_{g}$ that dependent on finite value of quark chemical potential for flavor $N_{f}=3$. We study the total photon spectrum over the space-time evolution of QGP.

In Figure [1], we plot production rate of photon with transverse momentum for various value of phenomenological parameter range $\gamma_{q}=2 \gamma_{g}$ to $8 \gamma_{g}$ with fix value of quark chemical potential $\mu_{q}=0.57 \mathrm{GeV}$ at thermal temperature $T_{0}=0.57 \mathrm{GeV}$ for flavor $N_{f}=3$. We found that the photon production rate strongly increases with increases the quark phenomenological parameter at quark chemical potential $\mu_{q}=0.57 \mathrm{GeV}$ and at higher temperature $T_{0}=0.57 \mathrm{GeV}$. Also a uniform fall is observed in total LO emission rate as a function of transverse momentum $k_{T}$ for all values of phenomenological parameter. The increase in the emission rate is highly effected by baryon rich plasma of the system with the various set of initial condition.

The total rate is significantly large and more dominant in the relevant range of transverse momentum. Also we observe that the photon yield supress more as the value of parameter $\gamma_{q}>$ 


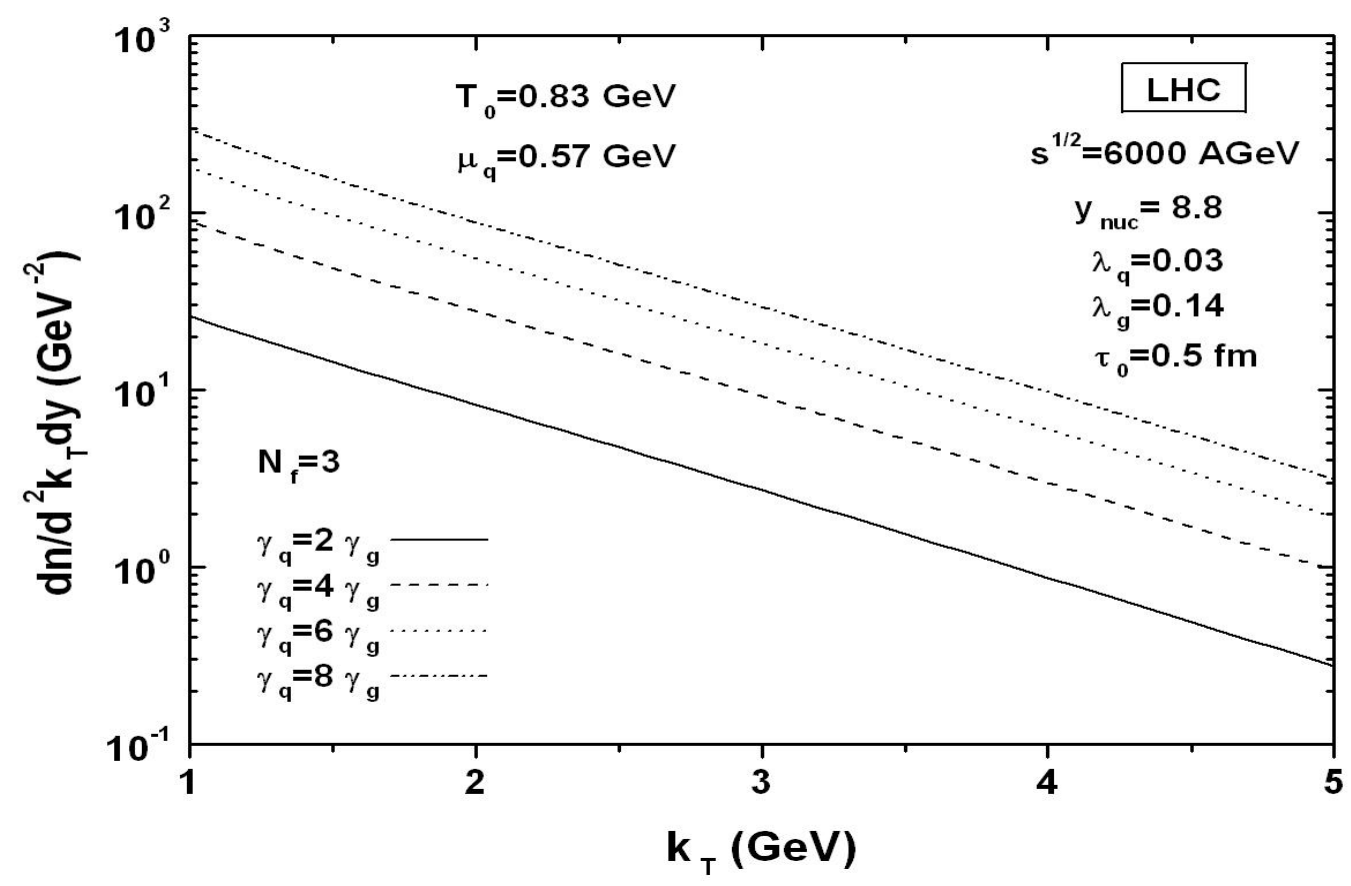

Figure 2: The photon spectra at thermal temperature $T_{0}=0.83 \mathrm{GeV}$ for $N_{f}=3$ with the finite value of quark chemical potential $\mu_{q}=0.57 \mathrm{GeV}$ with fix value of $\gamma_{g}=1 / 3$ and various value of parameter range from $\gamma_{q}^{\prime}=2\left[1+\mu_{q}^{2} / \pi^{2} T^{2}\right] \gamma_{g}$ to $8\left[1+\mu_{q}^{2} / \pi^{2} T^{2}\right] \gamma_{g}$ i.e. $\gamma_{q}=2 \gamma_{g}$ to $8 \gamma_{g}$ using HIJING initial condition at LHC.

$8 \gamma_{g}$. Thus, any higher values of quark phenomenological parameters are not suitably fit to get the complete pattern of photon spectra. At the same time, it is also not right to take low value of quark phenomenological parameter i.e. $\gamma_{q}<2 \gamma_{g}$ since for these values the calculation for photon rate diverges.

In Figure [2], we again set initial condition for LHC at higher temperature $T_{0}=0.83 \mathrm{GeV}$ and at fix value of quark chemical potential $\mu_{q}=0.57 \mathrm{GeV}$ for flavor $N_{f}=3$. It is found that the production rate of LHC data is much higher than the RHIC data. It implies that photon yield from the plasma at LHC leads higher production rate about a factor of $10^{1}$ to $10^{2}$ for flavor 3 .

Overall our model results are suitably valid for $\gamma_{q}=2 \gamma_{g}$ to $\gamma_{q}=8 \gamma_{g}$ with different set of parameter. One can observe that these factors enhance the contribution of the integration over the plasma evolution to the photon emission. At both RHIC and LHC energies, total photon yield of the system rises with the influence of quark phenomenological parameter and hence it is a strong increasing function at high temperature and chemical potential. We show the interesting results in the range of low and intermediate transverse momentum and found consistent output in the range $2 \gamma_{g} \leq \gamma_{q} \leq 8 \gamma_{g}$ to get complete picture of photon yield. Thus photons are regarded as potential probe of QGP in relativistic heavy-ion collisions. 


\section{Conclusion}

The production rate of photon from a chemically non-equilibrating system in heavy ion collision at RHIC and LHC energies is discussed with transverse momentum using various phenomenological parameter range from $\gamma_{q}=2 \gamma_{g}$ to $8 \gamma_{g}$ dependent on finite value of quark chemical potential for flavor $N_{f}=3$. It provides a good opportunity to study the evolution of fireball in high-energy heavy-ion collisions. The consideration of flow parameters dependent on quark chemical potential in quark mass show the important role in the photon measurements. The outcome of photon production as a function of transverse momentum incorporating the parametrization factors from $\gamma_{q}=2 \gamma_{g}$ to $\gamma_{q}=8 \gamma_{g}$ give new improved results. Also these results are more enhanced as comparison to the results of Ref. [35, 40] at zero chemical potential. Our model results for photon measurement from a chemically non-equilibrating plasma may provide more information for understanding the properties of QGP in the field of high energy heavy ion collisions.

\section{Acknowledgments}

We would like to thank D. S. Gosain for helpful discussions in preparing the manuscript.

\section{References}

[1] A. Andronic, P. Braun-Munzinger, and J. Stachel, Nucl. Phys. A 772 (2006) 167.

[2] I. Arsene, et al., (BRAHMS Collaboration), Nucl. Phys. A 757 (2005) 1; B. B. Back, et al., (PHOBOS Collaboration), Nucl. Phys. A 757 (2005) 28.

[3] K. Adcox, et al., (PHENIX Collaboration), Nucl. Phys. A 757 (2005) 184; J. Adams, et al., (STAR Collaboration), Nucl. Phys. A 757 (2005) 102.

[4] A. Adare, et al., (PHENIX Collaboration), Phys. Rev. C 81 (2010) 034911; S. S. Adler, et al., (PHENIX Collaboration), Phys. Rev. Lett. 98 (2007) 012002; A. Adare, et al., (PHENIX Collaboration), Phys. Rev. Lett. 98 (2007) 162301.

[5] C. Adler, et al., (STAR Collaboration), ibid. 89 (2002) 092301; T. Chujo (PHENIX Collaboration), Nucl. Phys. A 715 (2003) 151c.

[6] R. Chatterjee, E.S. Frodermann, U.W. Heinz and D.K. Srivastava, Phys. Rev. Lett. 96 (2006) 202302.

[7] J. Rafelski, B. Muller, Phys. Rev. Lett. 48 (1982) 1066; E. Shuryak, Phys. Rep. 80 (1980) 71.

[8] K. Kajantie, J. Kapusta, L. McLerran, A. Mekjian, Phys. Rev. D 34 (1986) 2746. A. Dumitru, D.H. Rischke, Th. Schonfeld, et al., Phys. Rev. Lett. 70 (1993) 2860.

[9] S. Turbide, C. Gale, S. Jeon and G.D. Moore, Phys. Rev. C 72 (2005) 014906.

[10] J. Kapusta, L.D. Mclerran and D.K. Srivastava, Phys. Lett. B 283 (1992) 145.

[11] T. Renk, Phys. Rev. C 74 (2006) 034906.

[12] M.M. Aggarwal et al., (WA98 Collaboration), Phys. Rev. Lett. 85 (2000) 3595; Nucl-ex/0006007; (WA98 Collaboration), Phys. Rev. Lett. 93 (2004) 022301; R. Albrecht, et al., Phys. Rev. Lett. 76 (1996) 3506.

[13] S.S. Adler et al., (PHENIX Collaboration), Phys. Rev. Lett. 94 (2005) 232301; D.P. Morrison, (PHENIX Collaboration), Nucl. Phys. A 638 (1998) 565c. 
[14] R. Baier, H. Nakkagawa, A. Neigawa and K. Redlich, Z. Phys. C 53 (1992) 433; M. Neubert, Z. Phys. C 42 (1989) 231.

[15] L. Mclerran and Toimela, Phys. Rev. D 31 (1985) 545.

[16] B. Sinha, Phys. Lett. B 128 (1983) 91; 157 (1985) 221; J. Alam, S. Sarkar, T. Hatsuda, T.K. Nayak and B. Sinha, Phys. Rev. C 63 (2001) 021901(R).

[17] C. Gale and J.I. Kapusta, Nucl. Phys. B 357 (1991) 65.

[18] J.D. Bjorken, Phys. Rev. D 27 (1983) 140.

[19] S. Nagamiya, Nucl. Phys. A 544 (1992) 5c.

[20] T.S. Biro, E.V. Doorn, B. Müller, M.H. Thomas and X.N. Wang, Phys. Rev. C 48 (1993) 1275.

[21] L. Xiong and E. Shuryak, Phys. Rev. C 49 (1994) 2203.

[22] X.N. Wang, Phys. Rep. 280 (1997) 287.

[23] C.T. Traxler and M.H. Thoma, Phys. Rev. C 53 (1996) 1348.

[24] M. Strickland, Phys. Lett. B 331 (1994) 245.

[25] A. Dumitru et al., Mod. Phys. Lett. A 8 (1993) 1291.

[26] C.T. Traxler, H. Vija and M.H. Thoma, Phys. Lett. B 346 (1996) 329.

[27] Z. He, J. Long, Y. Ma and G. Ma, Chin. Phys. Lett. 22 (2005) 1350.

[28] D. Dutta, S.V.S. Sastry, A.K. Mohanty, K. Kumar and R.K. Choudhury, Pram. J. Phys. 60 (2003) 1079; D. Dutta, A.K. Mohanty, K. Kumar and R.K. Choudhury, Phys. Rev. C 61 (2000) 064911.

[29] J. Long, Z. He, Y. Ma and B. Liu, Nucl. Phys. A 766 (2006) 201; J. Long, Z. He, Y. Ma and B. Liu, Phys. Rev. C 72 (2005) 064907.

[30] H. Gervais and S. Jeon, Phys. Rev. C 86 (2012) 034904.

[31] A. Peshier, B. Kampfer, O.P. Pavlenko and G. Soff, Phys. Lett. B 337 (1994) 235; M.I. Gorenstein and S.N. Yang, Phys. Rev. D 52 (1995) 5206.

[32] R. Ramanathan, Y. Mathur, K.K. Gupta and A.K. Jha, Phys. Rev. C 70 (2004) 027903; S.S. Singh, D.S. Gosain, Y. Kumar and A.K. Jha, Pram. J. Phys. 74 (2010) 27.

[33] D.S. Gosain, S.S. Singh and A.K. Jha, Pram. J. Phys. 78 (2012) 719.

[34] Y. Kumar and S.S. Singh, Can. J. Phys. 90 (2012) 955; S.S. Singh and Y. Kumar, J. Mod. Phys. 4 (2013) 582.

[35] Y. Kumar and S.S. Singh, ISRN HEP 2013 (2013) 156747.

[36] A. Meng Zhao et al., Mod. Phys. Lett. A 25 (2010) 47; V.M. Bannur, Eur. Phys. J C 50 (2007) 629.

[37] P. Arnold, G.D. Moore and L.G. Yaffe, JHEP 0111 (2001) 057.

[38] P. Aurenche, F. Gelis, R. Kobes and H. Zaraket, Phys. Rev. D 58 (1998) 085003; P. Aurenche, F. Gelis and H. Zaraket, Phys. Rev. D 61 (2000) 116001; P. Aurenche, F. Gelis and H. Zaraket, Phys. Rev. D 62 (2000) 096012.

[39] P. Arnold, G.D. Moore and L.G. Yaffe, JHEP 0112 (2001) 009.

[40] T. Renk, Phys. Rev. C 67 (2003) 064901.

[41] F.D. Steffan and M.H. Thoma, Phys. Lett. B 510 (2001) 98. 
[42] T. Peitzmann and M.H. Thoma, Phys. Rep. 364 (2002) 175.

[43] S.S. Singh and Y. Kumar, Int. J. Mod. Phys. A 29 (2014) 1450110.

[44] S.S. Singh and Y. Kumar, JOP: Conf. Series 535 (2014) 012002.

[45] S.S. Singh and Y. Kumar, Int. J. Mod. Phys. A 30 (2015) 1550020. 